\title{
SATELLITE OBSERVATIONS OF SOLAR RADIO BURSTS*
}

\author{
R. G. Stone, H. H. Malitson, J. K. Alexander, and C. R. Somerlock \\ (NASA Goddard Space Flight Center, Greenbelt, Md., U.S.A.)
}

The second Advanced Technology Satellite (ATS-II) carries a radio-astronomy experiment designed to perform radio-noise measurements in the range 0.5 to $3.0 \mathrm{MHz}$ from above the terrestrial ionosphere. The spacecraft was launched into an $11000-\mathrm{km}$ apogee, 180-km perigee orbit on April 6, 1967, and since there was considerable solar activity in the ensuing months it is possible to present a preliminary description of the kind of solar observations this experiment will provide.

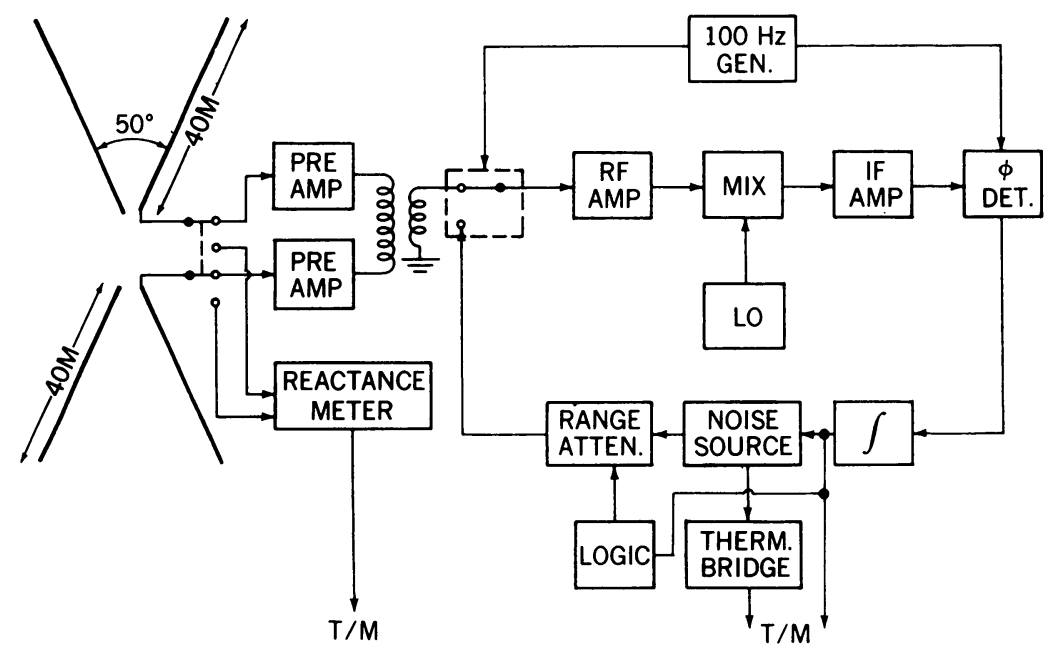

FIG. 1. Block diagram of ATS-II radio-astronomy experiment.

The essential features of the radio-astronomy experiment are shown in block diagram form in Figure 1. The satellite carries four 40-m booms in an X-configuration for a gravity-gradient stabilization experiment. One pair of these booms is used to form a dipole antenna which is a half-wavelength at about $1.8 \mathrm{MHz}$. The dipole is connected through a pair of high impedance pre-amplifiers to a Ryle-Vonberg type radiometer. The radiometer consists of a receiver which is switched between the

* Presented by M. R. Kundu.

Kiepenheuer (ed.), Structure and Development of Solar Active Regions, 585-587. () I.A.U. 
antenna and a reference-noise source at $100 \mathrm{~Hz}$. The difference between the antenna signal and the noise-source signal is synchronously detected to provide an error signal which adjusts the noise-source output to match the antenna signal. The noise-source output, which is then equal to the noise power from the antenna, is measured in a precision thermistor bridge and telemetered to the ground. The system has an effective bandwidth of $40 \mathrm{kHz}$ and an integration time constant of about $1 \mathrm{sec}$. The radiometer steps through seven frequencies $-0 \cdot 45,0 \cdot 7,0 \cdot 9,1 \cdot 1,1 \cdot 6,2 \cdot 2$, and $3.0 \mathrm{MHz}-$ in $40 \mathrm{sec}$. Once during each 40 -sec cycle the antenna is connected to a reactance meter to provide a measurement of the antenna capacitance. Since the antenna is shared with a second experiment on the satellite, radio-noise measurements are obtained for a period of $10 \mathrm{~min}$, and then the experiment is off during alternate 10-min periods.

Numerous solar radio bursts have been observed with the ATS-II experiment, and although data analysis is still in progress we can describe phenomena being seen in a preliminary fashion. Figure 2 shows two type-III bursts observed on May 20, 1967. The first was observed in the frequency range 41 to $20 \mathrm{MHz}$ at $2028 \mathrm{UT}$, and the second from 41 to $27 \mathrm{MHz}$ at $2214 \mathrm{UT}$, with the University of Colorado radio spectrograph at Boulder, Colo., U.S.A. Associated with these ground-based obser-

(a)

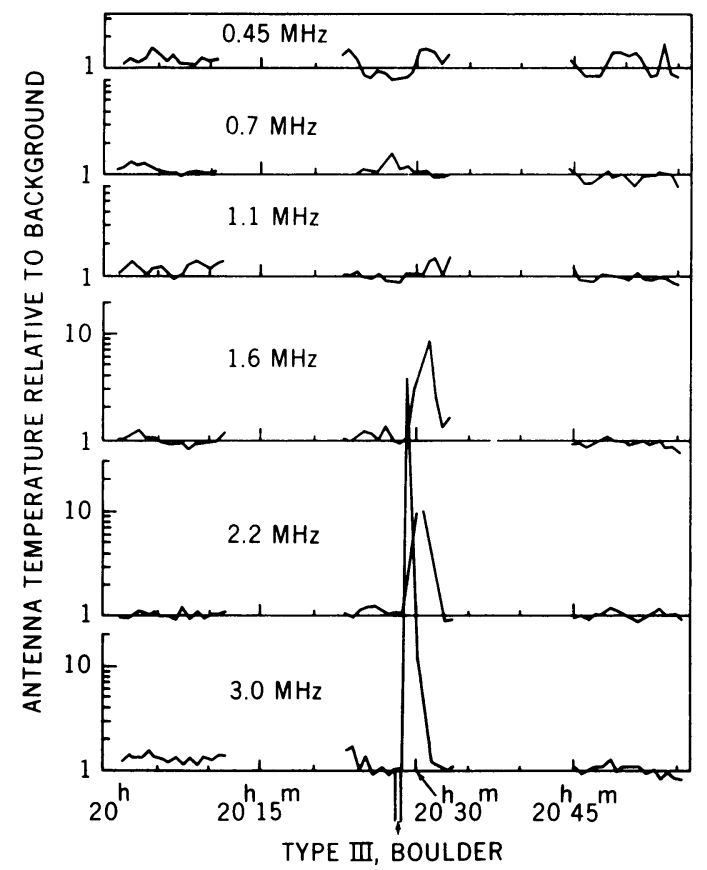

(b)

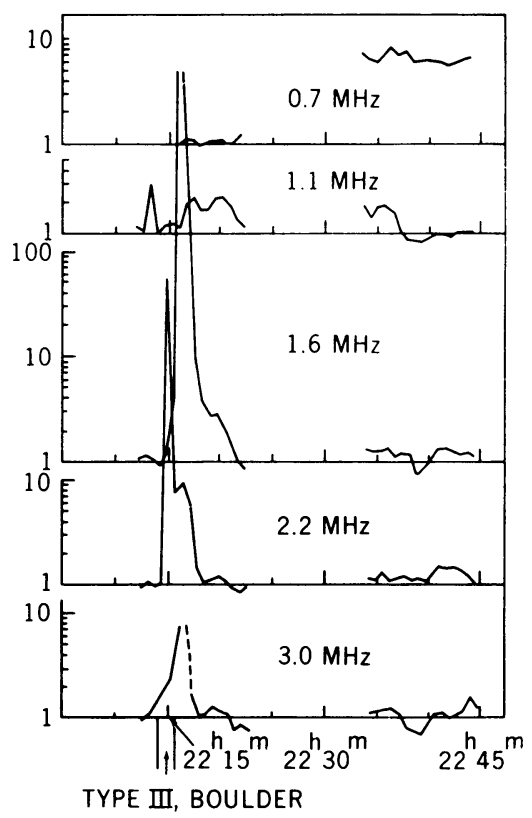

UNIVERSAL TIME, MAY 20, 1967

FIG. 2. Examples of Type-III solar radio bursts observed at low frequencies. 
vations, one can see abrupt enhancements in the low-frequency noise levels down to $1 \mathrm{MHz}$. If the type-III bursts are generated by moving disturbances which trigger radiation at the local electron-plasma frequency, then we are observing phenomena out to the order of 30 solar radii. By measuring the time of occurrence of the burst at each frequency and noting the time delay for the burst to occur at lower frequencies, one can deduce the velocity of the disturbance in the corona. For a reasonable model of the coronal electron-density distribution we get velocities which are typically $0 \cdot 1-0 \cdot 3 \mathrm{c}$. From the burst durations at each frequency we can estimate the temperature distribution in the outer corona. If one assumes that electron collisions are the principal mechanism for the decay, we find temperatures the order of $2 \times 10^{5} \mathrm{~K}$ at $15 R_{\odot}$. Of course, these numbers are derived from a very small sample of measurements; when analysis of a larger data sample is completed we can expect a much more reliable picture of the interaction of energetic particle streams with the outer corona.

On several occasions we have observed intense noise bursts associated with complex radio events including bursts of types III, II and IV observed on the ground. In such cases the noise level has been found to exceed the background level by at least 3 orders of magnitude from $3 \mathrm{MHz}$ down to at least $0.7 \mathrm{MHz}$. If one assumes an apparent source diameter of $1^{\circ}$ at $1 \mathrm{MHz}$, then this implies an equivalent brightness temperature in excess of $10^{15} \mathrm{~K}$. Such intense non-thermal radio bursts appear to be associated with the initial phases of complex, energetic, flare-associated events. 